\title{
Leadership Competency Model-Drenica: Generalizability of Leadership Competencies
}

\author{
Fadil Çitaku $^{1 *}$,Hetem Ramadani \\ ${ }^{1}$ Academy of Leadership Sciences Switzerland, Switzerland \\ ${ }^{2}$ Health Revolution, Albania
}

\section{Keywords:}

Leadership Competencies,

Model Drenica,

Leadership Generalizability

\section{Received}

18 September 2020

Received in revised form

22 September 2020

Accepted

22 September 2020

*Correspondence:

info@alss-edu.ch

ABSTRACT

This paper aims to summarize the generalizability of leadership competencies published in field of leadership. This present study used the Leadership Competencies Model of Çitaku et al. (2012), which has investigated six most developed western countries (Austria (A), Germany (D), Switzerland (CH), United States of America (USA), Canada (CA) and United Kingdom (UK)) to find out leadership competencies for efficient leaders. We randomly selected 25 leadership competencies using the Research Randomizer tool and systematically researched on Google Scholar, how many times occur leadership competencies, published between the 2010 and 2020. The analysis of the related literature was conducted by employing a bibliometric analysis of the research on the Google Scholar database between 2010 and 2020. The results of present study showed that there is an outstanding interest in our randomly chosen leadership competences and that these 25 competencies can be applied in any leadership domain. The study brought out 25 important leadership competencies incorporated in seven important domains, resulting with leadership competencies model named DRENICA, which is scientifically robust and can be used in leadership training courses and programs, in all leadership domains. The study concluded that 25 randomly chosen leadership competencies are robust, generalized and can be used in any leadership domain. Future research should be designed to replicate, extend and confirm the present findings. Meanwhile, we have provided a "Leadership Competency Model-Drenica" that can be employed to teach and further investigate leadership competences.

CCIKD Publishing

According to Bennis (2007), an expert in the study of leadership, an important threat facing the world today is the lack of effective leadership of our human institutions. Indeed, Lipman-Blumen (2006) has called attention to the failure of leadership in government, universities, healthcare and financial institutions. Organisations need competent and effective leaders now more than ever to face the threats and challenges of the modern world (Bisbee, 2007; Zaccaro, 2007). 
Long-time scholars in the field of leadership (Vroom \& Jago, 2007) defined leadership as a 'process of motivating people to work together collaboratively to accomplish great things' (p. 18). Accordingly, leadership is a process, not a property of a person. It involves a particular form of influence called motivating, resulting in collaboration in pursuit of a common goal to achieve the great things that are in the minds of both leader and followers (Vroom \& Jago, 2007).

\section{Leadership Theories}

In a recent meta-analysis of trait and behavioural theories of leadership (Derue, Nahrgang, Wellman, \& Humphrey, 2011) concluded that much of the research evidence fails to provide an integrated framework for understanding what constitutes leadership effectiveness. They did empirically identify some leader traits and behaviours that represent effective leadership. The concept of leadership overlaps with two similar terms, management and administration. The former is used widely in Europe and Africa, while the latter is preferred in the USA, Canada and Australia. Leadership is often of great contemporary interest in most countries in the developed world (Bush, 2003).

Some leadership researchers (Bush, 2003; Spillane, Halverson, \& Diamond, 2004) distinguish between leadership, administration and management. They suggest that leadership is synonymous with change, while management and administration are considered as maintenance. All three dimensions are identified as critical functions of organizational activity. Taken together, leadership can be construed as a means of shaping the goals, motivations and actions of others to initiate change or maintain stability (Bush, 2007). Some researchers (Bush, 2007; Spillane et al., 2004) have adopted a social perspective to conceptualize leadership. Spillane et al. (2004) for example, argued that leadership activity is defined or constructed through the interaction of leaders and followers during the execution of leadership tasks.

\section{The Importance of Effective Leadership}

Leadership is a complex multifaceted phenomenon that is widely observed but poorly understood. Many authors (Avolio et al., 2009; Minvielle, 1997; Schwartz \& Pogge, 2000; Taylor, Taylor, \& Stoller, 2008; Waters \& Grubb, 2004; Wharton, 1987) have argued that high-quality leadership is imperative to the success of organizations. Many researchers (Taylor, Taylor, \& Stoller, 2008; Waters \& Grubb, 2004; Wharton, 1987) have emphasized idealized personal characteristics such as educational visionaries, instructional and curriculum leaders, assessment experts, community builders, public relations experts, budget analysts, facility managers, special programmes administrators and expert overseers of legal, contractual and policy mandates, and initiatives are thought to characterize effective leaders. The preponderance of empirical evidence, however, does not support this trait model of leadership effectiveness (Derue et al., 2011). Although effective leaders can have a positive influence on achievement, poor leaders can have a marginal or even negative impact on success (Waters \& Grubb, 2004).

Waters and Grubb (2004) in their study reported three major findings that support the notion that school-level leadership matters in student achievement. First, they found that principal leadership was correlated with student achievement; one SD improvement in principal leadership was associated with a 10 percentile increase in student achievement. Second, they identified several leadership practices or processes required to fulfil a number of responsibilities that were significantly and directly related to student achievement. Third, they found a differential impact of 
leadership_ just as leaders can have a positive impact on student's achievement, they also can have marginal or, worse, a negative impact on student's achievement.

While the need for effective leaders is widely acknowledged, there is much less certainty about which leadership behaviours are most likely to produce favourable outcomes (Bush, 2007). In consonance with the conclusions of others (Bennis, 2007; Çitaku, 2012; Derue et al., 2011) — the foregoing review indicates that further empirical work in leadership is required. Given that leadership is associated with employee achievement, successful team functioning and efficient institutional operations, it is critical that an empirically supported comprehensive definition be developed.

\section{Leadership Competencies}

Although there is no commonly agreed upon definition of a general theory of leadership, leadership has been pragmatically defined as a process of motivating people to achieve great things. However, defining the required competencies for effective leadership is complex and poorly understood. One innovative study on this subject was performed by Çitaku et al. (2012) in which a carefully validated questionnaire of 63 items were sent to the key leaders in six countries: Austria, Canada, Germany, Switzerland, UK and USA. This study received a large number of responses and uncovered interesting variations in the valuation of specific leadership competencies. This study also revealed interesting differences in the valuation of specific competencies depending on the sex, native language, and area of specialization of the respondent, especially for the domains of social responsibility, innovation, and justice orientation.

Çitaku et al.'s (2012) study is considered important because the authors proposed a tool that enables an objective and standardized assessment of perceptions on leadership competencies. Furthermore, this tool was specifically developed to be applied in the health care setting. Measuring leadership competencies is important to guide and tailor the training of current and aspiring leaders. Since the publication of this landmark study, limited research has been performed specifically in the field leadership. Çitaku et al.'s (2012) study indicates that core competencies in leadership can be empirically identified and categorized into five factors: (1) Social Responsibility, (2) Innovation, (3) Self-Management, (4) Task Management, and (5) Justice Orientation that are theoretically meaningful, coherent, internal consistent and parsimonious in explaining the variance of the data. Although there are some between-group differences in the factors, there are no substantive differences by country or language (English vs German language). Accordingly, the competencies appear to be stable and coherent.

Other research outcomes (Eisenberger, 2015; Rock, D., 2008) that promise a big improvement in leadership are the outcomes from the neuroscience, emphasizing the importance of brain knowledge for leaders. For example, the importance of social responsibility as a key factor in leadership. These outcomes correlate very well with Çitaku's Leadership Competency Model since this model at the very top includes the leadership domain social responsibility.

\section{Methods}

This study, the research of generalizability of leadership competencies has used as a research tool, the Çitaku's Model of Leadership Competencies (2012), which has scientifically investigated six countries (A, D, CH, USA, CA, UK) to find out leadership competencies in Medical and Healthcare Education. Since Çitaku's study was focused on Medical and Healthcare Education only, we wanted to find out to what extent 25 out of 63 leadership competencies of this model can show 
generalizability and can be applied generally in all domains of leadership. For this purpose, we first checked in Google how many times the five domains (1) Social responsibility 2) Innovation Selfmanagement 4) Task management 5) Justice orientation) of Çitaku's leadership competencies model occur in general. After this we randomly, using the Research Randomizer tool, selected 25 leadership competencies and systematically researched on Google Scholar, how many times these 25 leadership competencies occur between 2010 and 2020. The analysis of the related results was conducted by employing a bibliometric analysis of the research on the Google Scholar database between 2010 and 2020. The results of present study showed that there is an outstanding interest in our randomly chosen leadership competences and that these 25 competencies can be applied in any leadership domain

\section{Data Analysis Procedures and Results}

We started our analysis by searching on Google for keyword "Leadership 2010-2020". The Google showed us 12'900'000 hits. Then we combined keywords/phrases "Leadership Competencies 2010-2020“. This time the google showed us these results 494'000. Afterwards, we wanted to focus more on scientific results of the leadership theories and used Çitaku's (2012) Leadership Competency Model (which has investigated leadership competencies in six western countries and cultures: Austria, Germany, Switzerland, United States of America, Canada and United Kingdom, and has been replicated and retested in other cultures). We were searching on Google for the domains (phrases) that incorporates this model between 2010 and 2020. The Table 1 shows the results:

Table 1

Domains of Leadership Competencies based model of Çitaku et al., between 2010 and 2020, Google search

\begin{tabular}{ll}
\hline Domains of Leadership Competencies 2010-2020 & Results on Google 2010-2020 \\
\hline Social responsibility and leadership & $434^{\prime} 000$ \\
Innovation and leadership & $1^{\prime} 270^{\prime} 000$ \\
Self-management and leadership & $353^{\prime} 000$ \\
Task management and leadership & $467^{\prime} 000$ \\
Justice orientation and leadership & $402^{\prime} 000$ \\
\hline
\end{tabular}

1) "Social responsibility and leadership 2010-2020", the google showed 434'000 hits. 2) “Innovation and leadership 2010-2020", the google showed 1'270'000 hits. 3) "Self-management and leadership", the google showed 353'000 results. 4) "Task management and leadership 20102020", the google showed 467'000 hits. 5) “Justice orientation and leadership 210-2020", the google showed 402 '000 hits.

In the next step we randomly, using the Research Randomizer tool, selected 25 leadership competencies and systematically researched on Google Scholar, how many times occur these leadership competencies published between 2010 and 2020.

For the 25 items (leadership competencies) on purpose we didn't re-test them statistically, since they were very deeply statistically proven by the previous study (Çitaku et al., 2012). The means of the items ranged from 3.85 to 4.76. The SDs were typical $(<1.0)$ for five-point items, indicating that data points are clustered closely around the mean (Çitaku et al., 2012). We found out that 25 competencies (words/phrases), randomly chosen from Çitaku's (2012) study occur in Google Scholar between the period 2010 and 2020, as shown in Table 2. 
Table 2

Represents 25 Competencies (words/phrases), from Çitaku's Model. (2012) found on Google Scholar, Published between 2010 and 2020

\begin{tabular}{|c|c|c|}
\hline Most common leadership competencies on Google Scholar & All Results on Google Scholar & Results on Google Scholar \\
\hline 1. $\quad$ Dignity and respect & 1’130’000 & 3’100 \\
\hline 2. Distributing rewards fairly & $81^{\prime} 400$ & 3’230 \\
\hline 3. Decision Making & 2’230’000 & $39^{\prime} 000$ \\
\hline 4. Reflection & 4'600’000 & $23 ’ 900$ \\
\hline 5. Relationship building & $5{ }^{\prime} 8500^{\prime} 000$ & $33 ’ 500$ \\
\hline 6. Responsibility for others & 3’950’000 & $27^{\prime} 700$ \\
\hline 7. Reinforcing change & $1 ’ 550 ’ 000$ & $17 ’ 300$ \\
\hline 8. Ethics & 4’350’000 & $17^{\prime} 600$ \\
\hline 9. Enhancing task knowledge & 3’980’000 & $19^{\prime} 600$ \\
\hline 10. Eliminating barriers to performance & $4400^{\prime} 000$ & $14^{\prime} 400$ \\
\hline 11. Evaluating consequences & 5’070’000 & $24 ’ 700$ \\
\hline 12. Explaining decisions with respect & 2’330’000 & $19^{\prime} 800$ \\
\hline 13. Empowerment & $2 ’ 280 ’ 000$ & $6 ’ 550$ \\
\hline 14. Nurturing relationships & $332^{\prime} 000$ & 3'810 \\
\hline 15. Integrity and Honesty & $272^{\prime} 000$ & 1’070 \\
\hline 16. Identifying problems & 4'850’000 & $29^{\prime} 400$ \\
\hline 17. Intelligent risk taking & 3’510’000 & $17^{\prime} 600$ \\
\hline 18. Communication with community & 4’710’000 & $27 ’ 500$ \\
\hline 19. Continuous learning & $5 ’ 250 ’ 000$ & $222^{\prime} 500$ \\
\hline 20. Critical thinking & 4'590’000 & $19^{\prime} 500$ \\
\hline 21. Creative problem solving & $2 ’ 520 ’ 000$ & $18^{\prime} 100$ \\
\hline 22. Collaborating & $936^{\prime} 000$ & $18 ’ 100$ \\
\hline 23. Active listening & $2 ’ 860 ’ 000$ & 5,330 \\
\hline 24. Adaptability & 1'380’000 & $6 ’ 020$ \\
\hline 25. Achieve goals & 4’390’000 & $36 ’ 300$ \\
\hline
\end{tabular}

The final step of our study was to comprise these competencies in one leadership model and to give to the model a name that has a meaning and serves as donkey bridge. After many times sorting out the first letters of each leadership competency, and trying different names, we have decided to name it "Leadership Competency Model Drenica" (LCMD) as presented in Table 3. This name fits well, since incorporates all 25 leadership competencies randomly selected. It is a coincidence, but also pride that both authors of this study were born in a region of Kosova, called Drenica.

Table 3

Leadership Competency Model Drenica (LCMD)

\begin{tabular}{|l|l|}
\hline $\mathbf{D}$ & Dignity and respect; Distributing rewards fairly; Decision Making \\
\hline $\mathbf{R}$ & Reflect; Relationship building; Responsibility for others; Reinforcing change \\
\hline $\mathbf{E}$ & $\begin{array}{l}\text { Ethics; Enhancing task knowledge; Eliminating barriers to performance; Evaluating consequences; Explaining decisions with } \\
\text { respect }\end{array}$ \\
\hline $\mathbf{N}$ & Nurturing relationships \\
\hline $\mathbf{I}$ & Integrity and Honesty; Identifying problems; Intelligent risk taking \\
\hline $\mathbf{C}$ & Communication with community; Continuous learning; Critical thinking; Creative problem solving; Collaborating \\
\hline $\mathbf{A}$ & Active listening; Adaptability; Achieving goals \\
\hline
\end{tabular}




\section{Discussion}

The purpose of the present study was to identify from the literature the most effective competencies of leadership published in Google Scholar. The model is based on a list of 63 leadership competencies identified in the previous research Çitaku's Model of Leadership Competencies (2012). This study re-tested the importance of the five domain of leadership competencies: Social Responsibility, Innovation, Self-Management, Task Management and Justice Orientation. Cronbach's alpha reliability of the factors were high, tested in the previous study, Çitaku et al. (2012), indicated good coherence and internal consistency.

These combined in present study suggest that these 25 leadership competences can be used generalized, represent a coherent, reliable and parsimonious model of leadership and show the application in all domains of leadership.

\section{Conclusion}

The present study re-tested core competencies in leadership that can be identified and categorised into five domains: (1) Social Responsibility, (2) Innovation, (3) Self-Management, (4) Task Management, and (5) Justice Orientation, similarly as in the study of Çitaku et al. (2012). The present study confirmed that these 25 leadership competencies possess generalizability, are theoretically meaningful, coherent and internal consistent. Notwithstanding the limitations of the present study, it is one of the few that has explicitly defined and provided evidence for leadership competencies considered to be the most important in leadership science. By testing the generalizability of 25 leadership competencies we have managed to develop the "Leadership Competency Model-Drenica", which can be applied in any leadership domain and any culture. Future research should be designed to replicate, extend and confirm the present findings. Meanwhile, we have provided a model that can be employed to teach and further investigate leadership competences.

\section{References}

Avolio, B. J., Reichard, R. G., Hannah, S. T., Walumbwa, F. O., Chan, A., Reichard, R. G., Hannah, S. T., \& Walumbwa, F. O. (2009). A meta-analytic review of leadership impact research: Experimental and quasi-experimental studies, The Leadership Quarterly, 20(5), 764-784.

Bennis, W. (2007). The challenge of leadership in the modern world: Introduction to special issue. American Psychologist ,62, 25.

Bisbee, D. C. (2007). Looking for leaders: Current practices in leadership identification in higher education. Planning and Changing, 38, 77-88.

Bush, T. (2003). Theories of educational leadership and management. Thousand Oaks, CA: SAGE Publications Ltd.

Bush, T. (2007). Educational leadership and management: Theory, policy and practice. South African Journal of Education, 27(3), 391-406.

Çitaku, F., Violato, C., Beran, T., Donnon, T., Hecker, K., \& Cawthorpe, D. (2012). Leadership competencies for medical education and healthcare professions: population-based study. BMJ Open, 2: e000812. http://dx.doi.org/10.1136/bmjopen2012-000812

Derue, D. S., Nahrgang, J. D., Wellman, N. E. D., \& Humphrey, S. E. (2011). Trait and behavioural theories of leadership: An integration and meta-analytic test of their relative validity. Personnel Psychology, 64(1),7-52.

Eisenberger, N. I. (2015). Social pain and the brain: Controversies, questions, and where to go from here. Annual Review of Psychology, 66, 601-629.

Lipman-Blumen, J. (2006). The allure of toxic leaders: Why we follow destructive bosses and corrupt politicians-and how we can survive them. New York: Oxford University Press. 
Minvielle, E. (1997). Beyond quality management methods: Meeting the challenges of health care reform. International Journal for Quality in Health Care, 9(3), 189-192.

Rock, D. (2008). SCARF: a brain-based model for collaborating with and influencing others. Neuro Leadership Journal, 1(1), 44-52.

Spillane, J. P., Halverson, R., Diamond, J. B. (2004). Towards a theory of leadership practice: a distributed perspective. Journal of Curriculum Studies, 36(1), 3-34.

Schwartz, R., \& Pogge, C. (2000). Physician leadership is essential to the survival of teaching hospitals. The American Journal of Surgery, 179(6), 462-468.

Taylor, C. A., Taylor, J. C., \& Stoller, J. K. (2008). Exploring leadership competencies in established and aspiring physician leaders: An interview-based study. Journal of General Internal Medicine, 23(6), 748-754.

Waters, T., \& Grubb, S. (2004). The leadership we need: Using research to strengthen the use of standards for administrator preparation and licensure programs. Aurora, CO: Mid-continent Research for Education and Learning.

Vroom, V. H., \& Jago, A. G. (2007). The role of the situation in leadership. American Psychologist, 62(1),17-24.

Wharton, C. R. (1987). Leadership in medical education: the challenge of diversity. Academic Medicine, 62(2), 86-94.

Zaccaro, S. J. (2007). Trait-based perspectives of leadership. American Psychologist, 62(1), 6-16. 